\title{
PARAMETERS OF THE PLANTING MACHINE FOR SUGAR BEET SETS
}

\author{
V.V.Mikheev ${ }^{\mathrm{a}}$, M. Szymanek ${ }^{\mathrm{b}}$, W . Tanas ${ }^{\mathrm{c}^{*}}$, P.A. Eremin ${ }^{\mathrm{a}}$, V.A. Kolos ${ }^{\mathrm{a}}$, V.B. Lovkis ${ }^{\mathrm{d}}$ \\ ${ }^{a}$ Federal Research Agro-engineering Center VIM, Moscow, Russian Federation, \\ e-mail: miheev-vim@mail.ru \\ ${ }^{\mathrm{b}}$ Department of Agricultural, Forest and Transport Machinery, University of Life Sciences in Lublin, \\ Poland, e-mail: mariusz.szymanek @up.lublin.pl, ORCID: 0000-0002-3337-0337 \\ ${ }^{\mathrm{c}}$ Department of Agricultural, Forest and Transport Machinery, University of Life Sciences in Lublin, \\ Poland, e-mail: wojciech.tanas@up.lublin.pl, ORCID: 0000-0002-9544-8902 \\ d Belarussian State Agrarian Technical University, Minsk, Republic Belarus, \\ e-mail: vlovkis@batu.edu.by \\ ${ }^{*}$ Corresponding author: e-mail: wojciech.tanas@up.lublin.pl
}

\begin{tabular}{|c|c|}
\hline ARTICLE INFO & ABSTRACT \\
\hline $\begin{array}{l}\text { Article history: } \\
\text { Received: November } 2019 \\
\text { Received in the revised form: } \\
\text { December } 2019 \\
\text { Accepted: December } 2019\end{array}$ & $\begin{array}{l}\text { For seed production of sugar and fodder beet, the creation of machines } \\
\text { and working bodies for planting root crops on testes and pollinators is } \\
\text { of current importance. It was found out that the seed-and-water produc- } \\
\text { tion was spreading with the use of "schteklings" [sugar beet roots]. In } \\
\text { the countries with minus temperatures between the vegetation seasons }\end{array}$ \\
\hline $\begin{array}{l}\text { Key words: } \\
\text { scheme, } \\
\text { parameter, } \\
\text { apparatus, } \\
\text { schtekling, } \\
\text { landing, } \\
\text { beet }\end{array}$ & $\begin{array}{l}\text { it is necessary to dig out beets and plant them again at the beginning of } \\
\text { the vegetation season. Advantages and disadvantages of machines and } \\
\text { apparatus for disembarking the schteklings were determined. Under the } \\
\text { conditions of applicability, we chose a design scheme and a type of } \\
\text { landing gear. With the use of mathematical expressions and standards } \\
\text { for the labor intensity of the operator of the above machine, the optimal } \\
\text { parameters of its planting apparatus were determined. It was determined } \\
\text { that the work of the operator of the schteklings planting machine's stem } \\
\text { will meet the safety standards for the intensity of labor if the planting } \\
\text { unit of this machine is a rotor-type. It should be equipped with planting } \\
\text { cones and a charging device, a conveyor type. The number of holders } \\
\text { of landing cones should be } 6-8 \text { pieces, and the radius of the rotor is } \\
\text { within the range of } 0.344 \ldots . .382 \mathrm{~m} \text {. Such a scheme, type and parame- } \\
\text { ters of the landing gear will allow operators to work at the speed of the } \\
\text { planter of up to } 1.2 \mathrm{~m} \cdot \mathrm{s}^{-1} \text {. }\end{array}$ \\
\hline
\end{tabular}

\section{Введение}

До недавнего времени в нашей стране были распространены толь-ко две схемы семеноводства сахарной и кормовой свеклы - высадочная и безвысадочная (Mikheev et al., 2016). Первая схема по данным семеноводов затратная, а вторая - имеет ограниченное распространение по зонам - на Кубани и в Крыму. Анализ показал, что в других странах получают распространение штеклинговая, рассадно-пересадочная, многолетняя и др. технологии (Apasov, 2017; Zharkov, 1996). Так, в ведущих 
компаниях Германии - KWS, Франции - Florimond Despres, Бельгии - Ses Vanderhave, CШA - Betaseed.INC (шт. Миннесота) по семеноводству сахарной свеклы кроме высадочных и многолетних, используются также безвысадочные (в Африке, Италии) и безвысадочно-пересадочные (штеклинговая) технологии, последняя из которых осваивается и в России.

Если в технологическом плане штеклинговая технология, как приоритетное направление развития семеноводства в нашей стране нашло практическую индустриальную поддержку, в лице ООО «Щелково Агрохим», то с точки зрения ее технического обеспечения нет эффективных решений по конструкциям машин и обоснованию их параметров для почвообработки, высадки штеклингов и их уборке. Критической является операция высадка штеклингов.

Цель исследований - обоснование схемы и параметров высадкопосадочного аппарата для штеклингов свеклы.

Материалы и методы. Как показали исследования, для высадки маточников корнеплодов свеклы культур (штеклингов) или рассады используют универсальные машины (придаются как опции).

Ранее, в СССР были разработаны высадкопосадочные машины ВПС-2,8 и полуавтоматическая ВПС-2,8А. Эти машины значительно превосходили зарубежные аналоги по производительности и уменьшению затрат труда на посадке, однако их выпуск уже прекращен. По нашему мнению, они имели и недостатки - значительную энергоемкость, т.к. в них использовались бороздообразователи, что требовало применения трактора класса 3, а также они не пригодны для высадки штеклингов.

В настоящее время ФГБНУ ВНИИСС разработаны проект технологии для выращивания семян свеклы из штеклингов и опытный образец высадкопосадочная машина ВШ-4 (www.vniiss.110mb.com). Эти разработки только проходят опытную апробацию и достоверных данных о ее завершении не имеется.

Маркетинговый анализ показал, что на рынке доступны и другие машины с инновационными высаживающими аппаратами. Это аппараты следующих типов (www.agro-sistema) (Рис. 1):

- вертикальным - обеспечивает очень бережную высадку, так как стаканчик с рассадой плавно опускается на цепной передаче/или диске до самой поверхности почвы и только тогда открывается - машины типа Over, DUE MANUAL, A1 (Герм.);

- револьверным - характеризуется высокой производительностью и удобством загрузки рассады оператором - машина фирмы IMAC (Итал.). Но в этой машине большая высота падения высаживаемых растений;

- с зажимами - предназначен для высадки рассады/корня с «голыми» корневищами машина Basrijs BY (Англ.). 
Parameters of the planting...

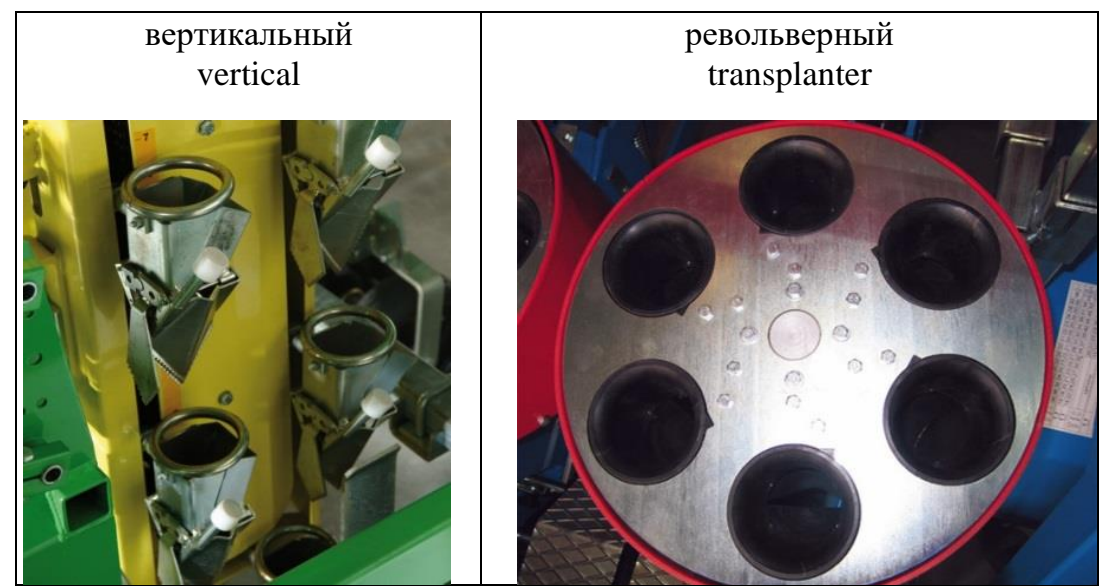

Рисунок 1. Типь высаживающих аппаратов

Figure 1. Types of planting units

В машине типа Over отличительной особенностью является отсутствие сошников на высаживающих элементах, благодаря чему высадка рассады может быть проведена как по открытому, так и по уже профрезерованному грунту, путем вдавливания конуса в рыхлую почву, с последующим раскрытием его створок, укладкой корней/рассады в лунку, закрытием почвой и уплотнением катками. Высаживающий элемент машины конус. Он прикреплен к ротору (Рис. 2). Однако в этой машине создаваемое ложе (лунка) не увлажняется перед высадкой культуры, что ухудшает ее приживаемость.

В Германии разработана аналогичная машина, но только для посадки свекловичных штеклингов и других корнеплодов под маркой А1. Однако этой машине присущ тот же недостаток, что и предыдущей и, кроме того, требуется четкая синхронизация работы сажальщика и высаживающего аппарата, позволяющая работать на больших скоростях от существующей $\left(0,3-0,5 \mathrm{M} \cdot \mathrm{c}^{-1}\right)$.

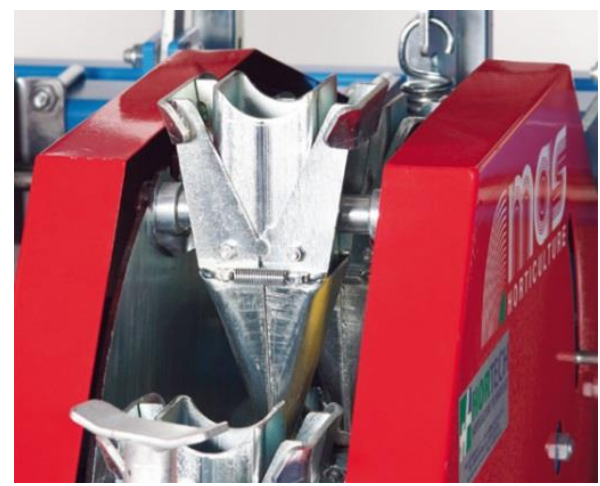

Рисунок 2. Высаживающий аппарат в виде конуса машины Over Figure 2. Planting unit in the form of the Over machine cone 
В силу специфики ручных работ при подаче штеклингов в высаживающий аппарат, вызванной тем, что они имеют значительно меньшие размерные и массовые характеристики чем ранее использовавшиеся маточные корнеплоды свеклы, то в настоящее время трудно установить наиболее приемлемый рабочий орган штеклинго посадочной машины, не проведя аналитическую оценку его работоспособности и удовлетворению существующим нормам напряженности труда сажальщиков.

Результаты и обсуждение. Анализ показал, что наибольшее распространение имеют полуавтоматические штеклинго посадочные машины, с ручной подачей штеклингов в высаживающий аппарат роторного или ленточного типов (Рис.3) (Mikheev et al., 2016; Izmailov and Lobachevsky, 2012).

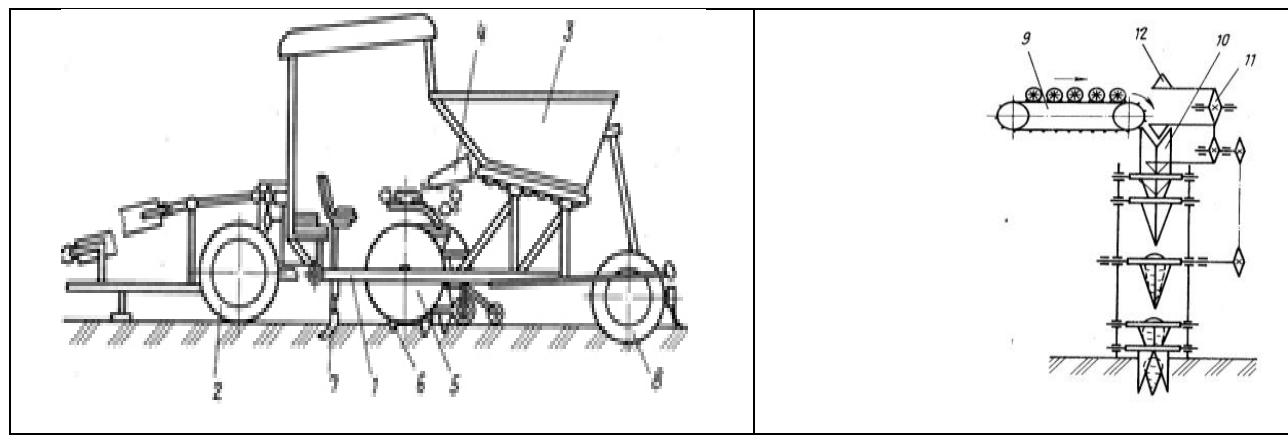

a)

б)

Рисунок 3. Конструктивная схема машины для посадки штеклингов: а - вид сбоку; б - высаживающий аппарат

Figure 3. Structural scheme of a schtecling seeding machine: $a$-side view; $b$-planting unit

Машина состоит из рамы 1 с опорными колесами 2, бункера 3 , лотков-накопителей 4, зарядного устройства, роторных аппаратов 5 с посадочными конусами 6, рыхлителей 7 (устанавливаются при работе на не подготовленной почве) и прикатывающих колес 8 .

Зарядное устройство машины включает транспортер 9, расположенный перпендикулярно к плоскости вращения роторного посадочного аппарата 5 с посадочными конусами 6, лоток $10 \mathrm{~V}$-образной формы, расположенного в плоскости вращения роторного аппарата 5. Лоток 10 имеет переменную кривизну по длине, приближаясь своим концом к окружности вращения посадочных конусов 6. Над лотком 10 установлены дополнительный транспортер 11, снабженный лопастями 12 $\mathrm{V}$-образной формы с размерами, равными размерам лотка 10 , и планки-успокоители 13 и 14, закрепленных с помощью шарнира 15.

Планка-успокоитель 13 установлена в зоне изменения кривизны лотка 10, а одна из планок-успокоителей 14 установлена над концом лотка 10. Приводы транспортеров 9 и 11 зарядного устройства и посадочного аппарата 5 кинематически связаны и синхронизированы, что упрощает труд оператора. 
Parameters of the planting...

Предлагаемая схема высаживающего аппарата машины позволяет улучшить качество посадки корнеплодов и повысить производительность зарядного устройства, если оптимизированы его параметры по условиям:

1. интенсивность укладки штеклинга оператором (при ручной подаче) в посадочный аппарат, роторный или ленточный должна быть нормативная ГОСТ Р 53489-2009:

- нормативная Иа $\leq 30 \ldots 40$, шт мин $^{-1}$,

- опытная (ВИМ) Иа $\leq 1 \ldots 2, \quad$ шт $\cdot \mathrm{c}^{-1}$,

2. густота посадки штеклингов в почву задана агротребованиями (Mikheev et al., 2016; Zharkov, 1996):

- при междурядьях 45-60 см Гш $=4 \ldots 5$ шт $\mathbf{M}^{-1}$,

- при междурядьях $70 \mathrm{~cm}$ Гш $=3 \ldots 4$ шт $\cdot \mathrm{M}^{-1}$.

3. допустимая рабочая скорость машины определяится из соотношения:

$$
\mathbf{V p}=\text { Иа: ГшI , м } \cdot \mathrm{c}^{-1}
$$

4. период времени между ручной последовательной закладкой штеклингов в держатели/конусы высаживающего аппарата, имеющего радиус по концам держателей $\mathbf{R}$ и их число $\mathbf{Z}$ составит:

$$
T_{3}=2 \pi R /(Z \omega),
$$

Где

T3 - интервал закладки штеклингов в аппарат, с

$\mathbf{R}$ - радиус высаживающего аппарата по местам закладки, м

$\mathbf{Z}$ - число держателей/конусов штеклингов на аппарате, шт

$\boldsymbol{\omega}$ - линейная скорость держателей высаживающего аппарата по точкам закладки штеклингов, $\mathrm{m}^{-1}$

5. при известном шаге посадки число корнедержателей/конусов выбран с учетом показателя кинематической характеристики работы посадочной машины (8), представляющего собой отношение линейной скорости аппарата к поступательной скорости агрегата:

$$
\mathbf{\gamma}=\boldsymbol{\omega} / \mathbf{V p} \geq \mathbf{1}(\mathbf{\gamma} \leq 1,1),
$$

На практике, выдерживание условия равенства линейной скорости посадочного ротора и поступательной скорости машины трудно осуществимо, поэтому зачение $\mathbf{\gamma}$ принимают больше единицы, - на величину буксования приводных колес машины (до $10 \%$ - по данным испытаний).

Расчеты показали, что радиус высаживающего аппарата $\mathbf{R}$ может составлять до $0,573 \mathrm{M}$, при рабочей скорости до $1,2 \mathrm{M} \cdot \mathrm{c}^{-1}$ (табл.). С учетом условий вписываемости посадочного аппарата в габариты машины и условий работы операторов по технике безопасности (см. условие 1), радиус высаживающего аппарата должен находится в пределах не более $0,344 \ldots 0,382 \mathrm{M}$, а число конусов/корнедержателей, соответственно 6 - 8 шт.

Предложенные схема, тип и параметры посадочного аппарата найдут применение при создании/выборе аппарата и машины для высадки штеклингов. 
Таблица 1.

Параметры высаживающего аппарата

Table 1.

Parameters of planting unit Tabela

\begin{tabular}{|c|c|c|c|c|c|}
\hline \multirow[t]{2}{*}{$\begin{array}{l}\text { Число дертелей на } \\
\text { роторе } \mathbf{Z} \text {,шт } \\
\text { Number of grips } \\
\text { in a rotor Z, pcs }\end{array}$} & \multicolumn{5}{|c|}{$\begin{array}{c}\text { Радиус высаживающего аппарата } R(R=\mathbf{Z} \omega \mathbf{T} 3 / 2 \pi) \text {, } \\
\text { м в зависимости от рабочей скорости машины, м/с } \\
\text { Radius of planting unit } \mathrm{R}(\mathrm{R}=\mathrm{Z} \omega \mathrm{T} / 2 \pi), \\
\mathrm{m} \text { in relations to the working speed of a unit m/s }\end{array}$} \\
\hline & 0,3 & 0,5 & 0,8 & 1,0 & 1,2 \\
\hline 4 & 0,057 & 0,095 & 0,152 & 0,191 & 0,229 \\
\hline 6 & 0,086 & 0,143 & 0,23 & 0,286 & 0,344 \\
\hline 8 & 0,114 & 0,191 & 0,305 & 0,382 & 0,458 \\
\hline 10 & 0,143 & 0,238 & 0,382 & 0,477 & 0,573 \\
\hline
\end{tabular}

\section{Выводы}

Обоснованы схема, тип и параметры высадкопосадочного аппарата для штеклингов сахарной свеклы роторного типа. Он должен оснащаться высаживающими конусами и зарядным устройством, транспортерного типа. Число держателей посадочных конусов должно составлять 6-8 шт., а радиус ротора находится в пределах 0,344...0,382 м. Такие схема, тип и параметры посадочного аппарата позволят работать операторам штеклинго посадочной машины на скорости до $1,2 \mathrm{M} \cdot \mathrm{c}^{-1}$.

\section{References}

Mikheev, V.V., Kusova, N.I, Savenkov, A.A/, Eremin, P.A. (2016). Develop critical machine technologies, processes, technical solutions for selection and seed production in the production of sugar and fodder beet. Scientific report on the subject 24.3.8, Moscow, VIM, 161-162.

Apasov, I.V. (2017). The development of selection and seed production of sugar beet in the Russian Federation for the period 2017-2020. At the All-Russian Council. select. 27.02.2017. Moscow, VIM.

Zharkov, Yu.V. (1996). Scientific bases of intensification of sugar beet seeds reproduction. Special 06.01.05 - Selection and seed-growing. The dissertation author's abstract on competition of a scientific degree of the doctor of agricultural sciences. Moscow, VNIISS.

Izmailov, A.Yu., Lobachevsky, J.P. (2012). Innovative mechanized technologies and automated technical systems for agriculture/Modernization of agricultural production on the basis of innovative machine technologies and automated systems. X11 Internationa Scientific-Technical Conferences, $1,31-44$.

www.vniiss.110mb.com. (accessed on 15.12.2019)

www.agro-sistema.ru/index.php? (accessed on 15.12.2019) 
Parameters of the planting...

\section{PARAMETRY ZESPOLU WYSADZAJĄCEGO DO WYSADEK BURAKA CUKROWEGO}

Streszczenie. Dla produkcji nasion buraków cukrowych i pastewnych aktualnie znaczenie ma stworzenie maszyny do wysadzania wysadek. W krajach, w których między sezonami wegetacyjnymi panują ujemne temperatury konieczne jest wykopanie buraków i ponowne ich posadzenie na początku kolejnego sezony wegetacyjnego. Zaobserwowano rozprzestrzenianie się technologii upraw nasiennych buraków wykorzystujących wysadki. Analizując warunki sadzenia wysadek wybrano konstrukcję i rodzaj aparatu wysadzającego. Z wykorzystaniem matematycznych wzorów i norm intensywności pracy operatora danej maszyny dokonano charakterystyki optymalnych parametrów aparatu wysadzającego. Aparat wysadzający powinien być wyposażony w stożki (uchwyty) do sadzenia i ładowarkę. Ilość stożków (uchwytów) do sadzenia powinna wynosić 6-8 sztuk, a promień wirnika w granicach $0,344 \ldots 0,382$ $\mathrm{m}$. Parametry te pozwolą pracować operatorowi sadzarki z prędkością do $1,2 \mathrm{~m} \cdot \mathrm{s}^{-1}$.

Słowa kluczowe: schemat, parametr, aparat, wysadki buraków, sadzenie, buraki 